Published in final edited form as:

J Am Chem Soc. 2016 July 13; 138(27): 8324-8327. doi:10.1021/jacs.6b02469.

\title{
A Remarkable Oxidative Cascade That Replaces the Riboflavin C8 Methyl with an Amino Group during Roseoflavin Biosynthesis
}

\author{
Isita Jhulki, Prem K. Chanani, Sameh H. Abdelwahed, and Tadhg P. Begley ${ }^{\star}$ \\ Department of Chemistry, Texas A\&M University, 3255 TAMU, College Station, Texas 77843, \\ United States
}

\begin{abstract}
Roseoflavin is a naturally occurring riboflavin analogue with antibiotic properties. It is biosynthesized from riboflavin in a reaction involving replacement of the C8 methyl with a dimethylamino group. Herein we report the identification of a flavin-dependent enzyme that converts flavin mononucleotide (FMN) and glutamate to 8-amino-FMN via the intermediacy of 8 formyl-FMN. A mechanistic proposal for this remarkable transformation is proposed.
\end{abstract}

Roseoflavin (3, 8-dimethylamino-riboflavin) is a red flavin antivitamin synthesized by Streptomyces davawensis and Streptomyces cinnabarinus. ${ }^{1,2}$ It is the only biosynthesized flavin analog with antibiotic activity and binds to the flavin mononucleotide (FMN) riboswitch, thus repressing riboflavin biosynthesis. ${ }^{3,4}$ Roseoflavin has been used in the screening of mutants for maximum riboflavin production because bacteria overproducing riboflavin are resistant to roseoflavin toxicity. ${ }^{5,6}$ Remarkably, roseoflavin is biosynthesized from riboflavin by replacing the C8 methyl with a dimethylamino group (Figure 1). ${ }^{7,8}$ This is an unprecedented transformation in flavoenzymology. The methyltransferase catalyzing the final step of this transformation has been characterized,,${ }^{9,10}$ and the biosynthetic gene cluster from $S$. davawensis has been cloned and sequenced. ${ }^{2}$ The mechanism of the methyl group replacement chemistry has not yet been elucidated and is the subject of this paper.

Based on a mechanistic model (vide infra), it was discovered that 8 -formyllumiflavin reacts with ammonia to form 8-aminolumiflavin in 30\% yield (Figure 2) (Jhulki, I. and Begley, T. unpublished). This reaction suggests that roseoflavin might be formed from 8 -formylriboflavin in a similar oxidative displacement reaction.

*Corresponding Author: begley@ chem.tamu.edu.

Notes

The authors declare the following competing financial interest(s): While this paper was under review, similar findings were reported by Professor Mack using genetics rather than a chemical approach to the identification of AFMN synthase. ${ }^{22}$ The papers differ in some of the details of the reconstitution and mechanistic analysis.

Supporting Information

The Supporting Information is available free of charge on the ACS Publications website at DOI: 10.1021/jacs.6b02469. Detailed experimental procedures for the syntheses and characterization of formyl-lumiflavin (4), formyl-FMN (7), amino-FMN (8), and the PFBHA-derivatized oxime standards; AFMN synthase overexpression and purification; enzymatic assays; H/D exchange experiment; co-elution experiment; measurement of oxygen consumption for formyl-FMN formation in AFMN synthase assay; NMR and LCMS analyses (PDF) 
Examination of the genes in the sequenced roseoflavin biosynthetic cluster from $S$. davawensis revealed that only one gene in this cluster was annotated as a flavoenzyme (BN159_7989, NADPH-dependent FMN reductase). We therefore proposed that Orf7989 might encode an 8-amino-flavin synthase.

Orf7989 was overexpressed in E. coli BL21(DE3) and purified on a nickel NTA affinity column. The purified protein was yellow, consistent with flavin binding. LC-MS analysis of the metabolites that copurified with Orf7989 revealed the presence of bound FMN, 8formyl-FMN and 8-amino-FMN (Figures 3 and 4). This observation suggested that Orf7989, heterologously expressed in the absence of all of the other roseoflavin biosynthetic enzymes, catalyzed the formation of 8-amino-FMN from FMN via the intermediacy of 8-formyl-FMN (Figure 5). Even though it is generally assumed that product dissociation rates are much faster than enzyme purification rates, we have previously found several other examples of enzymes that co-purify with product or intermediates and view this type of analysis as one of the key experiments for the identification of the reaction catalyzed by an enzyme of unknown function. ${ }^{11-13}$

To test the hypothesis shown in Figure 5, we identified formyl-FMN as the product formed when FMN was treated with amino-FMN (AFMN) synthase in the absence of the amine donor (Figures 6, 7, and 8). This reaction is oxygen requiring and does not occur under anaerobic conditions. The search for a suitable amine donor revealed that ammonia, pyridoxamine, tyrosine, serine, histidine, tryptophan, phenylalanine, methionine, arginine, lysine, and aspartate were not donors, glutamine was a weak donor, and glutamate was the best donor (Figures 6 and 7) and was converted to $a$-ketoglutarate (Figure 9).

A mechanistic proposal for this remarkable conversion is shown in Chart 1. In this proposal deprotonation of the $\mathrm{C} 8$ methyl group of FMN followed by oxidation gives the hydroperoxide $10 .{ }^{14}$ The $\mathrm{C} 8$ methyl group is known to be acidic, and this chemistry is also thought to occur during the covalent attachment of flavins to active site histidine and cysteine residues. ${ }^{15-17} \mathrm{Next}$, an active site base abstracts a $\mathrm{C} 8$ hydrogen to cleave the peroxide, leading to the formation of 8 -formyl-FMN 7. Amine addition to $\mathrm{C} 8$ followed by deformylation and air oxidation gives $\mathbf{2 1}$. Two successive tautomerizations give 23. Imine hydrolysis and air oxidation completes the formation of 8-amino-FMN 8.

This mechanistic proposal is consistent with the observed substrates and products of the reaction, with the intermediacy of formyl-FMN, and with the model system developed for the conversion of formyl-FMN to AFMN (Figure 2). We have also demonstrated that AFMN synthase catalyzes the deprotonation of the C8 methyl group of FMN (Figure S7), that the C8 carbon of FMN is released as formate (Figure S37), and that glutamic acid, the nitrogen source (labeling study in SI, Figure S4), is converted to $a$-ketoglutarate (Figure 9). No flavin modification is detected when the reaction is run in the absence of oxygen and glutamic acid, suggesting that 8-hydroxymethyl-FMN (13) is not an intermediate. This is further supported by demonstrating that hydrogen peroxide is not formed in the AFMN synthasecatalyzed oxidation of FMN to formyl-FMN (Figure S5 and S6). 
Thus, what initially appeared to be a highly complex reaction, requiring several enzymes, upon scrutiny turns out to be quite simple, involving an initial oxidation of the $\mathrm{C} 8$ methyl group to formyl-FMN followed by a four-electron oxidative cascade ${ }^{18-21}$ in which electrons are iteratively removed using the well-precedented oxidation of dihydroflavin by molecular oxygen. This study underscores the power of mechanistic reasoning to identify gene function. Mechanistic and structural studies are in progress to elucidate the details of this fascinating new riboflavin transformation.

\section{Supplementary Material}

Refer to Web version on PubMed Central for supplementary material.

\section{Acknowledgments}

This research was supported by the Robert A. Welch Foundation (A-0034 to TPB) and by a grant from the National Institutes of Health (DK44083).

\section{References}

1. Otani S, Takatsu M, Nakano M, Kasai S, Miura R. J Antibiot. 1974; 27:88.

2. Jankowitsch F, Schwarz J, Rueckert C, Gust B, Szczepanowski R, Blom J, Pelzer S, Kalinowski J, Mack M. J Bacteriol. 2012; 194:6818. [PubMed: 23043000]

3. Lee ER, Blount KF, Breaker RR. RNA Biol. 2009; 6:187. [PubMed: 19246992]

4. Langer S, Hashimoto M, Hobl B, Mathes T, Mack M. J Bacteriol. 2013; 195:4037. [PubMed: 23836860]

5. Perkins JB, Sloma A, Hermann T, Theriault K, Zachgo E, Erdenberger T, Hannett N, Chatterjee NP, Williams V II, Rufo GA Jr, Hatch R, Pero J. J Ind Microbiol Biotechnol. 1999; 22:8.

6. Russo P, Capozzi V, Arena MP, Spadaccino G, Duenas MT, Lopez P, Fiocco D, Spano G. Appl Microbiol Biotechnol. 2014; 98:3691. [PubMed: 24413973]

7. Pedrolli DB, Jankowitsch F, Schwarz J, Langer S, Nakanishi S, Mack M. Methods Mol Biol (N Y, NY, U S). 2014; 1146:41.

8. Matsui K, Juri N, Kubo Y, Kasai S. J Biochem. 1979; 86:167. [PubMed: 479119]

9. Jankowitsch F, Kuehm C, Kellner R, Kalinowski J, Pelzer S, Macheroux P, Mack M. J Biol Chem. 2011; 286:38275. [PubMed: 21911488]

10. Tongsook C, Uhl MK, Jankowitsch F, Mack M, Gruber K, Macheroux P. FEBS J. 2016; 283:1531. [PubMed: 26913589]

11. Chatterjee A, Abeydeera ND, Bale S, Pai P-J, Dorrestein Pieter C, Russell David H, Ealick Steven E, Begley Tadhg P. Nature. 2011; 478:542. [PubMed: 22031445]

12. Chatterjee A, Jurgenson CT, Schroeder FC, Ealick SE, Begley TP. J Am Chem Soc. 2006; 128:7158. [PubMed: 16734458]

13. Chatterjee A, Schroeder FC, Jurgenson CT, Ealick SE, Begley TP. J Am Chem Soc. 2008; 130:11394. [PubMed: 18652458]

14. Michel H, Hemmerich P. J Membr Biol. 1981; 60:143.

15. Jin J, Mazon H, van den Heuvel RHH, Heck AJ, Janssen DB, Fraaije MW. FEBS J. 2008; 275:5191. [PubMed: 18793324]

16. Hassan-Abdallah A, Zhao G, Schuman Jorns M. Biochemistry. 2008; 47:1136. [PubMed: 18179257]

17. Heuts DPHM, Scrutton NS, McIntire WS, Fraaije MW. FEBS J. 2009; 276:3405. [PubMed: 19438712]

18. Barry CE III, Nayar PG, Begley TP. J Am Chem Soc. 1988; 110:3333.

19. Dorrestein P, Begley TP. Bioorg Chem. 2005; 33:136. [PubMed: 15888307]

J Am Chem Soc. Author manuscript; available in PMC 2017 September 23. 
20. Dorrestein PC, Poole K, Begley TP. Org Lett. 2003; 5:2215. [PubMed: 12816412]

21. Maggio-Hall LA, Dorrestein PC, Escalante-Semerena JC, Begley TP. Org Lett. 2003; 5:2211. [PubMed: 12816411]

22. Schwarz J, Konjik V, Jankowitsch F, Sandhoff R, Mack M. Angew Chem, Int Ed. 2016; 55:6103. 

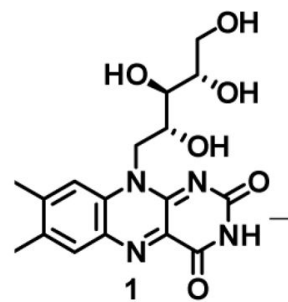

Riboflavin
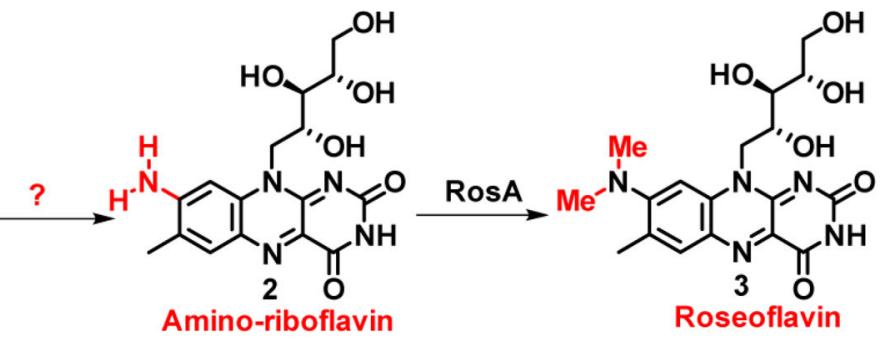

Figure 1.

Roseoflavin biosynthesis from riboflavin via amino-riboflavin in $S$. davawensis. 


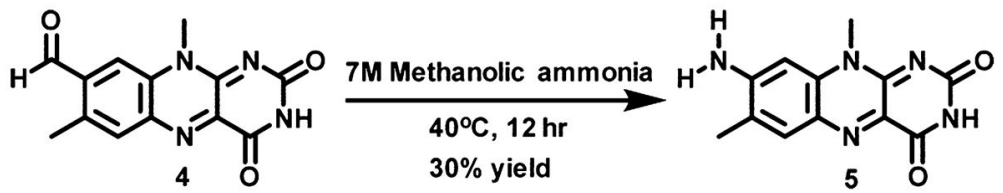

Figure 2.

Reaction of ammonia with 8-formyl-lumiflavin (4) to form 8-amino-lumiflavin (5). This reaction is a model system for the remarkable methyl group replacement that occurs during roseoflavin biosynthesis. 

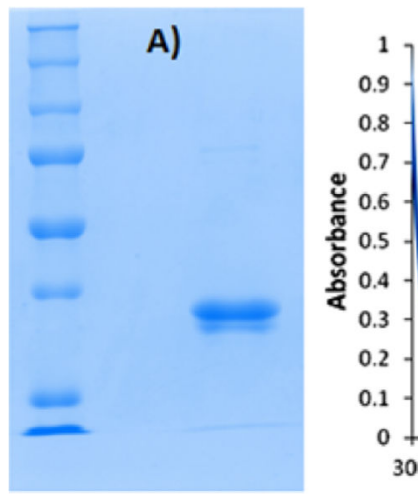

B)

)

Figure 3.

Analysis of the orf7989 encoded protein. (A) SDS-PAGE gel of the purified enzyme $(M \mathrm{~W}=$ $29 \mathrm{kDa}$ ). (B) UV-vis spectrum of Orf7989 showing an absorption maximum at $495 \mathrm{~nm}$ suggesting the presence of bound AFMN. 

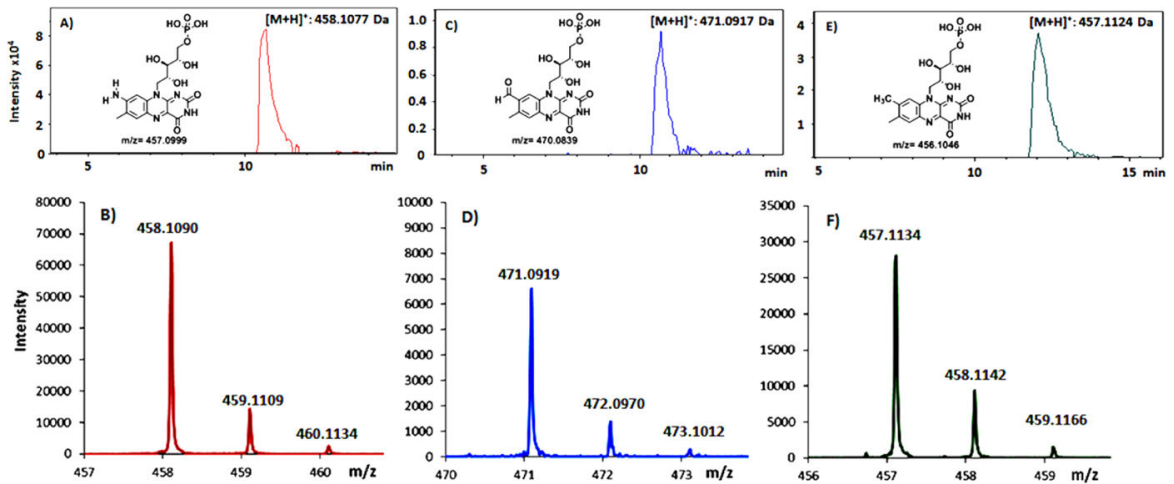

Figure 4.

Analysis of the denatured Orf7989 encoded enzyme showing that the protein copurified with AFMN, formyl-FMN and FMN. (A) Extracted ion chromatogram (EIC) at $m / z$ 458.1077.

(B) ESI-MS of the 458.1077 Da species consistent with AFMN. (C) EIC at $\mathrm{m} / z 471.0917$.

(D) ESI-MS of the 471.0917 Da species consistent with formyl-FMN. (E) EIC at $\mathrm{m} / z$ 457.1124. (F) ESI-MS of the 457.1124 Da species consistent with FMN. All spectra were obtained in positive ion mode. 
<smiles>[R]n1c2nc(=O)[nH]c(=O)c-2nc2cc(C)c(C)cc21</smiles>

$\mathrm{R}=$ Ribose phosphate<smiles>[Z]c1cc2nc3c(=O)[nH]c(=O)nc-3n([R])c2cc1C=O</smiles>

7<smiles>[R]n1c2nc(=O)[nH]c(=O)c-2nc2cc(C)c(N)cc21</smiles>

Figure 5.

Proposal for the AFMN synthase-catalyzed conversion of FMN to AFMN. 

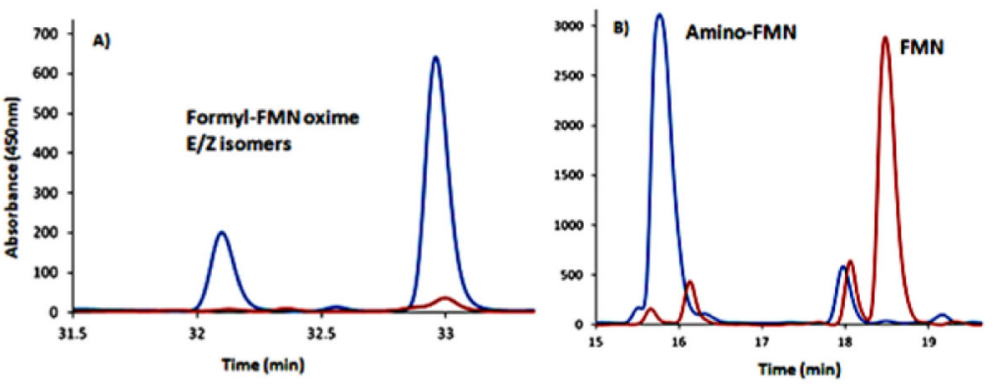

Figure 6.

AFMN synthase catalyzes the formation of formyl-FMN and AFMN in the absence and presence of glutamate. (A) HPLC analysis of the AFMN synthase-catalyzed oxidation of FMN followed by derivatization with pentafluorobenzyl hydroxylamine (PFBHA, blue trace, no glutamate and red trace, no enzyme control). The peaks eluting after 32.2 and 33 min correspond to the two PFBHA oxime isomers. (B) HPLC analysis of the AFMN synthase-catalyzed oxidation of FMN in the presence of glutamate (blue trace, full reaction and red trace, no enzyme conrol). 
A)
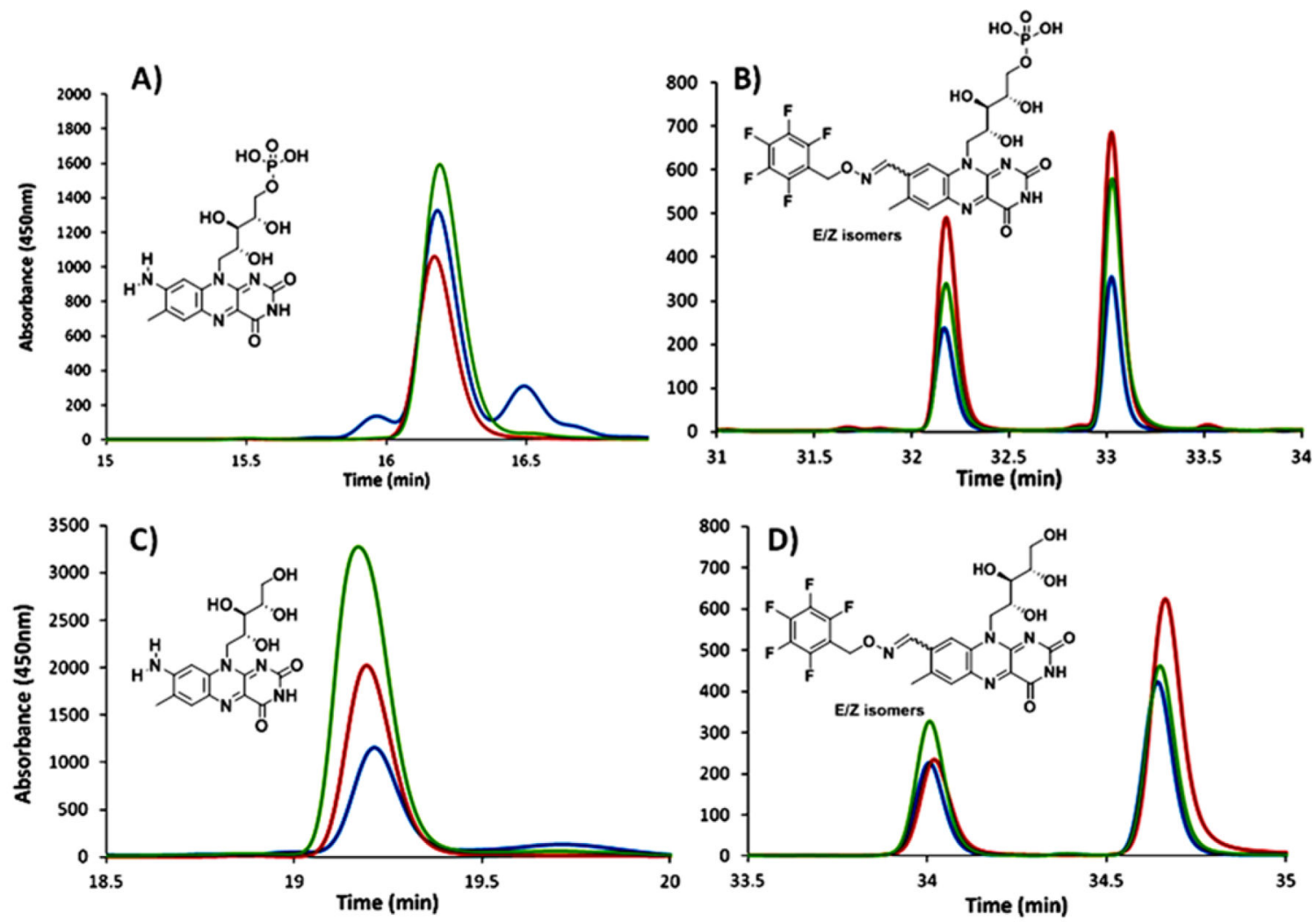

Figure 7.

Co-elution experiments with enzymatically formed formyl-FMN and AFMN and synthesized standards. (A) HPLC analysis of enzymatically formed AFMN (blue trace, enzymatically formed AFMN; red trace, AFMN standard; and green trace, co-elution of standard and enzymatic reaction mixture). (B) HPLC analysis of enzymatically formed formyl-FMN derivatized with pentafluorobenzyl hydroxylamine (blue trace, enzymatically formed formyl-FMN (absence of glutamate); red trace, formyl-FMN PFBHA oxime standard; and green trace, co-elution of standard and enzymatic reaction mixture after treatment with pentafluorobenzyl hydroxylamine). Diagrams (C and D) represent the same set of samples after phosphatase treatment showing comigration of amino-riboflavin and formyl-riboflavin PFBHA oxime with the dephosphorylated enzymatic products derivatized with PFBHA. 

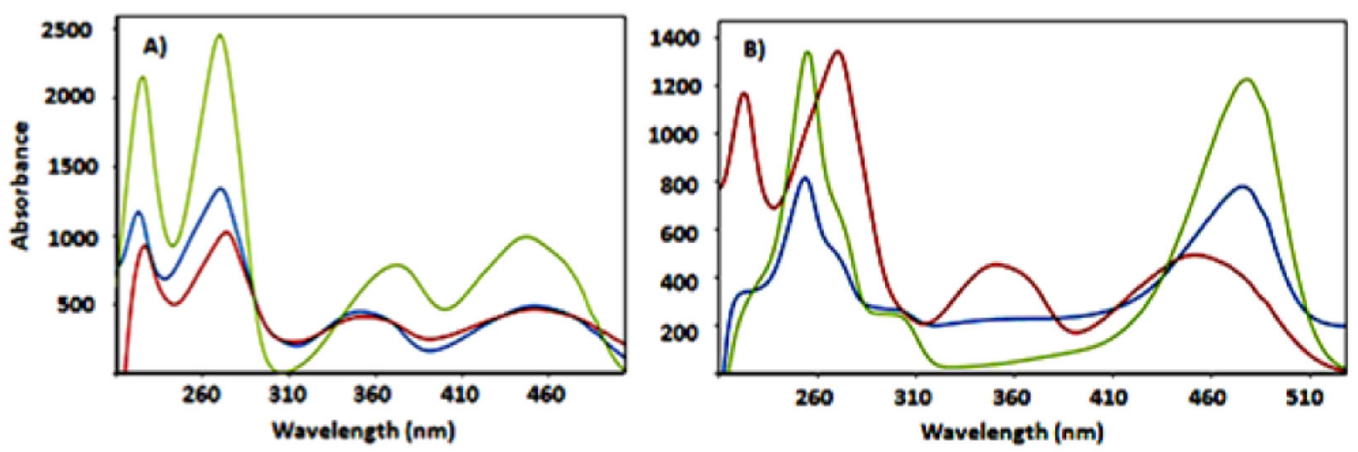

Figure 8.

Comparison of the UV-vis spectra of formyl and AFMN generated in the AFMN synthasecatalyzed reaction with authentic standards. (A) Overlay of the UV-vis spectra of enzymatically formed formyl-FMN (7, blue trace), formyl-lumiflavin (4, red trace), and FMN (6, green trace). (B) Overlay of the UV-vis spectra of enzymatically formed AFMN (8, green trace), formyl-FMN (7, red trace), amino-lumiflavin (5, blue trace). 

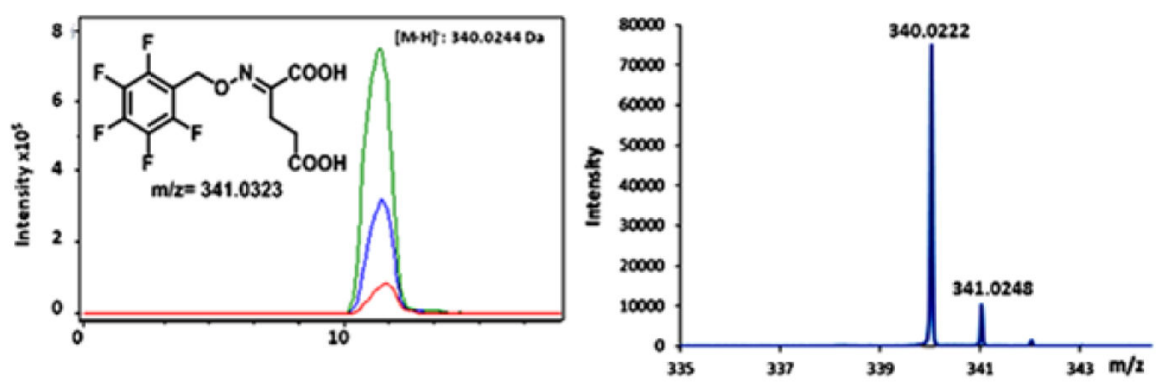

Figure 9.

LC-MS analysis of the $a$-ketoglutarate (24) PFBHA oxime generated from glutamate (17) in the AFMN synthase-catalyzed reaction (negative mode). Left, EIC at $\mathrm{m} / z 340.0244$ for the PFBHA trapped $a$-ketoglutarate (red trace, trapped $a$-KG formed in the enzymatic reaction; blue trace, trapped $a$-KG standard; and green trace, co-elution of trapped $a$-KG standard and same formed in the AFMN synthase-catalyzed reaction. Right, ESI-MS of the 340.0244 Da species consistent with the $a$-ketoglutarate PFBHA oxime. 


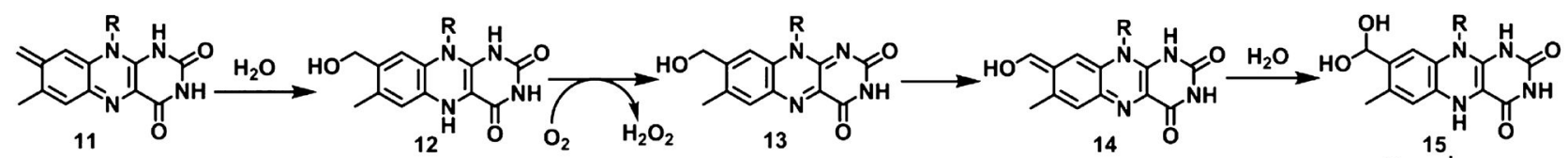
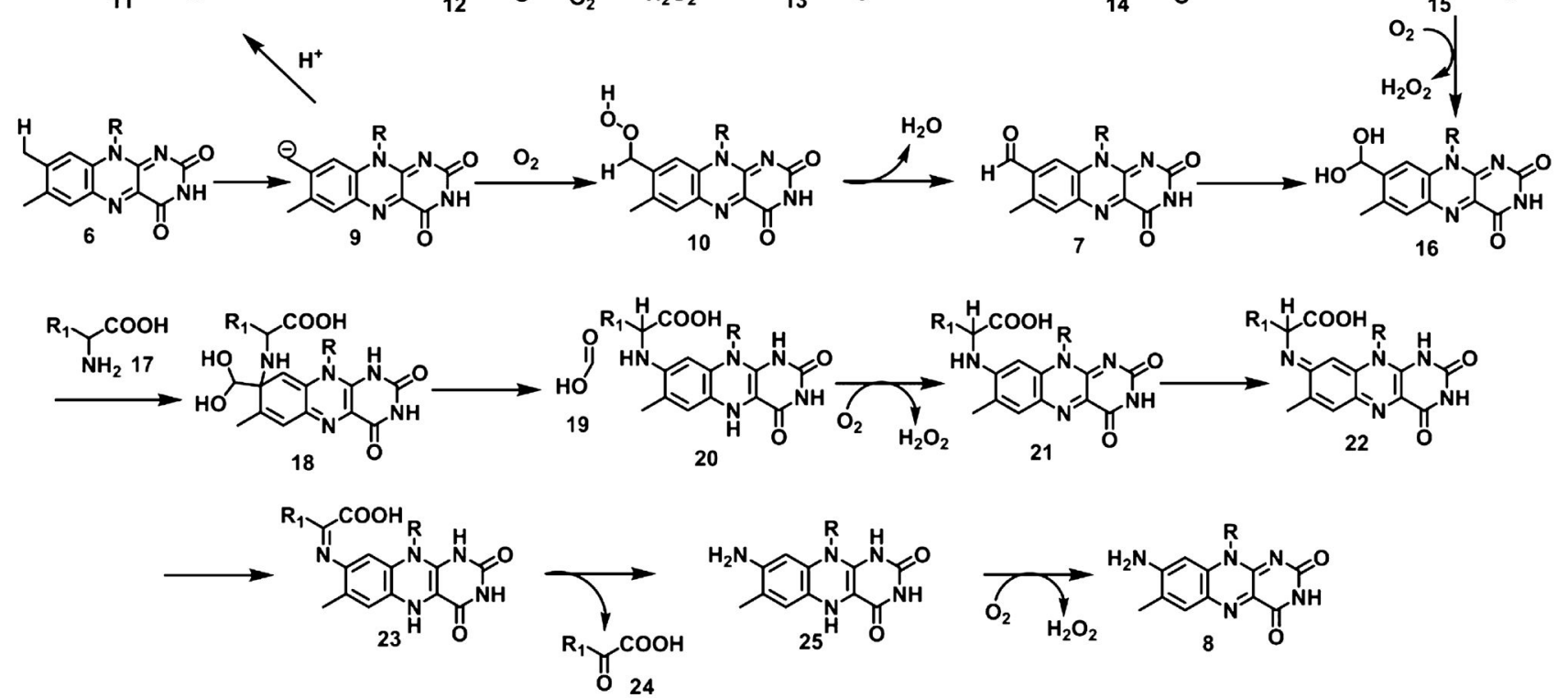

Chart 1.

Mechanistic Proposal for the AFMN Synthase-Catalyzed Reaction 